\title{
ERRATUM
}

\section{Manipulate light polarizations with metamaterials: From microwave to visible}

\author{
Jia-ming HAO (郝加明 $)^{1,2}$, Min QIU (仇旻 $)^{2}$, Lei ZHOU (周否 $)^{1, \dagger}$ \\ ${ }^{1}$ Surface Physics Laboratory (State Key Laboratory) and Physics Department, Fudan University, Shanghai 200433, China \\ ${ }^{2}$ Laboratory of Photonics and Microwave Engineering, School of Information and Communication \\ Technology, Royal Institute of Technology (KTH), Electrum 229, 164 40, Kista, Sweden \\ E-mail: ${ }^{\dagger}$ phzhou@fudan.edu.cn \\ Received July 21, 2010
}

Erratum to: Front. Phys. China, 2010, 5(3): 291-307

DOI 10.1007/s11467-010-0005-y

In page 300, Fig. (12) should be changed to

(a)
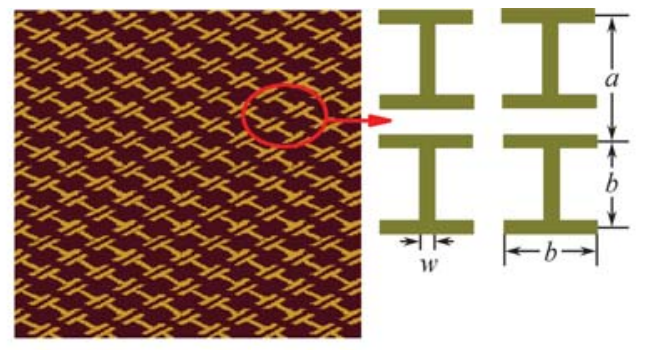

(b)

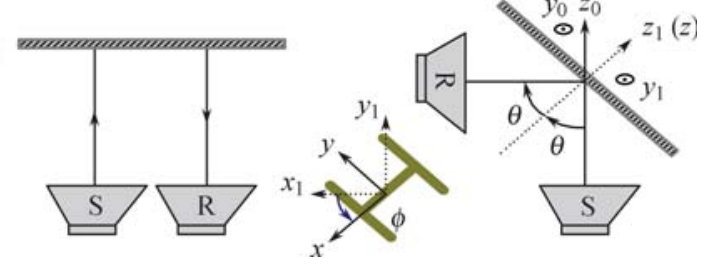

The online version of the original article can be found at http://dx.doi.org/10.1007/s11467-010-0005-y 